\title{
Rearrangeable $2 \times 2$ elastic optical switch with two connection rates and spectrum conversion capability
}

\author{
Wojciech Kabaciński ${ }^{1}$ (i) $\cdot$ Remigiusz Rajewski ${ }^{1}$ [ $\cdot$ Atyaf Al-Tameemi $^{1}$
}

Received: 10 March 2018 / Accepted: 8 July 2019 / Published online: 3 September 2019

(c) The Author(s) 2019

\begin{abstract}
In this study, we consider simultaneous connection routing in the three-stage switching fabric of the wavelength-spacewavelength architecture for elastic optical switches, which serve connections that can occupy different spectrum widths. Recently, the upper bound of the rearrangeability conditions was derived and proved for a switching fabric serving a limited number of connection rates. A control algorithm based on matrix decomposition was also proposed. In addition, the necessary and sufficient conditions were derived and proved for a switching fabric with a size of $2 \times 2$ serving only two connection rates, but only when the ratios between the connection rates and link capacity are integers. In this study, we extend these results to an arbitrary ratio between the connection rates and link capacity. The number of frequency slot units required in the interstage links is much lower than that in the strict-sense nonblocking switching fabrics. We also propose modifications to the control algorithm, which further reduces the number of frequency slot units required in many cases. Finally, we extend the results for $2 \times 2$ switching fabrics to those with a size of $r \times r$.
\end{abstract}

Keywords Elastic optical network $\cdot$ Elastic optical switching node $\cdot$ Interconnection network $\cdot$ Rearrangeable nonblocking condition

\section{Introduction}

In an elastic optical network, also known as flexible optical network, the spectrum is allocated to a lightpath according to the required spectrum and with certain granularity [1-3]. The smallest spectrum granularity is often called a Frequency Slot Unit (FSU). A spectrum assigned to one connection is called a frequency slot, and it may use $m$ adjacent FSUs. A connection that uses $m$ FSUs is called an $m$-slot connection. In the current standards, FSU has a width of $12.5 \mathrm{GHz}$ [4] but other values may be standardized in the future. The spectrum assigned to a lightpath depends on several factors, such as the required transmission speed,

Remigiusz Rajewski

remigiusz.rajewski@put.poznan.pl

Wojciech Kabaciński

wojciech.kabacinski@put.poznan.pl

Atyaf Al-Tameemi

atyaf.al-tameemi@doctorate.put.poznan.pl

1 Faculty of Electronics and Telecommunications, Poznan University of Technology, ul. Polanka 3, 60-965 Poznań, Poland distance that needs to be covered, path quality, wavelength spacing between channels, and/or the modulation scheme used $[2,3,5,6]$. In the current optical networks that mostly serve static traffic, the connections are known in the network design stage and the network can use only space switches without wavelength conversion. However, connections may change in time when the traffic changes from static to dynamic. Dynamic traffic results in spectrum fragmentation and a greater blocking probability [5].

The connections served in elastic optical network must be also served by switching nodes. Several architectures have been proposed for these switching nodes in previous studies [7-10], which were surveyed briefly [11]. One of these switching fabric architectures is the wavelength-space-wavelength (W-S-W) switching fabric [12], which is referred to as WSW1 [10]. This architecture is also considered in the present study.

The strict-sense nonblocking (SSNB) conditions (necessary and sufficient) for WSW1 were provided and proved in a previous study [12]. In SSNB switching fabrics, we can establish a connection from an idle set of FSUs at any input fiber to an idle set of FSUs at a requested output fiber regardless of how the other connections are established. However, 
the problem is that these SSNB switching fabrics require a huge number of FSUs in the interstage links, especially when the maximum number of FSUs that may be used by one connection is high.

SSNB switching fabrics usually require a large number of switching elements (crosspoints, centers stage switches, etc.), but this number can be reduced by applying rearrangements $[13,14]$. In rearrangeably nonblocking (RNB) switching fabrics, we can also connect any idle input and idle output pair, but it may be necessary to move the existing connections to alternate connecting paths. RNB switching fabrics are mostly used in packet switching, where the packets in synchronously operated (slotted) networks arrive at all inputs at the same time. A model describing these requests is called a simultaneous connection model and a set of requests is referred to as a set of compatible connections or simply a permutation $[13,14,16]$. The RNB conditions for space-division three-stage Clos switching fabrics [15] were derived in previous studies $[13,16]$. The RNB conditions for time-division switching networks with single-rate connections were considered by [17]. In addition, the RNB conditions for switching networks with multirate connections were obtained by [18].

In the case of elastic optical switches for the $\mathrm{W}-\mathrm{S}-\mathrm{W}$ architecture, two differences in the switching fabrics were considered by [18]: (1) a W-S-W switching fabric with no conversion capability but all of the switches are able to switch in time and space; and (2) connections with no adjacency constraints. According to [10], in the case of the SSNB conditions, the lack of conversion capability in the center stage switches and an adjacency constraint increases the required number of center stage switches or the number of FSUs in the interstage links. To the best of our knowledge, the RNB conditions for $\mathrm{W}-\mathrm{S}-\mathrm{W}$ switching fabrics for elastic optical nodes have only been considered in our recent previous study.

In [19], we first considered the upper bound for the RNB conditions of WSW1 switching fabrics, as well as providing the necessary and sufficient conditions for switching fabrics with two input and output fibers, and serving two connection rates. Another limitation is that the ratio between the connection rates as well as between the connection rates and the fiber capacity should be an integer.

In the present study, we extend our previously reported results [19] in three ways. First, for the connections considered in this study, we assume that the ratio between the connection rates and fiber capacity is not a whole number. This extension may appear simply theoretical, but this assumption is quite practical when we consider that although the guard-band is not needed between FSUs belonging to the same connection, the FSUs that are used by two different connections must be separated by the guard-band of 1 FSU, i.e., 1-slot and 2-slot connections become 2-slot and 3-slot connections, when this guard-band is included in the connection size. Elastic optical networks with two connection rates may be an attractive option for data center networks (DCNs), where traditional optical networks and switches currently allow reductions in power consumption [23] and latency [24]. For instance, the application of elastic optical networks in DCNs was considered by [24]. Second, we propose two different merging algorithms for reducing the number of FSUs required in the interstage links. Third, we show how the solution proposed for $2 \times 2$ switching fabrics can be used to control switching fabrics with a size of $r \times r$.

The reminder of this paper is organized as follows. In Sect. 2, we present the switching fabric and describe the problem. We also show why traditional simultaneous connection routing algorithms designed for three-stage Clos type switching fabrics cannot be used directly and modifications are required. In Sect. 3, we describe the model used in this study. We propose the matrix model for state representation and illustrate the basic characteristics and properties of this model. In Sect. 4, we present two control algorithms, which are based on merging permutation matrices. The first algorithm proposed in our previous study [19] and the second algorithm uses a different approach for merging permutation matrices. In Sect. 5, we derive and prove the sufficient conditions for the rearrangeability of $2 \times 2 \mathrm{WSW} 1$ switching fabrics for both control algorithms. We assume that this switching fabric serves two connection rates, but there are no restrictions on the ratios between the connection rates and fiber capacity. We also show how the results derived for the $2 \times 2$ switching fabric can be extended to switching fabrics with a size of $r \times r$. In Sect. 6, we compare the results obtained by using both algorithms in terms of the number of FSUs required in the interstage links. Finally, we give our conclusions.

\section{Problem statement}

In general, the WSW1 switching architecture has $r$ input and output fibers as described in detail by [12]. In the present study, we mainly consider switching fabrics where $r=2$, and we then show how the proposed algorithms can be used in case when $r>2$. An example of this type of switching fabric is shown in Fig. 1, where it comprises two bandwidthvariable wavelength converting switches (BV-WSs) in the first and third stages, and one bandwidth-variable wavelength selective space switch (BV-SS) with a capacity of $2 \times 2$ in the second stage. Each BV-WS in the first stage has one input fiber with $n$ FSUs ( $n=5$ in the example) and one output fiber with $k$ FSUs, whereas each BV-WS in the third stage has one input fiber with $k$ FSUs and one output fiber with $n$ FSUs. The internal architectures of the BV-WSs and BV-SS were described by [12]. The switching fabric serves 


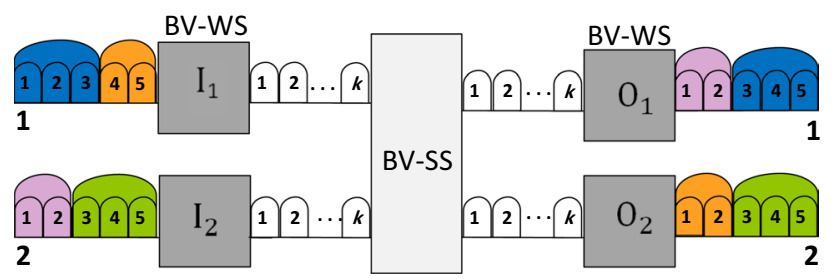

Fig. 1 WSW1 switching fabric with a set of connection requests, $\mathbb{C}=\left\{\left(I_{1}[1], O_{1}[3], 3\right) ;\left(I_{1}[4], O_{2}[1], 2\right) ;\left(I_{2}[1], O_{1}[1], 2\right) ;\left(I_{2}[3]\right.\right.$, $\left.\left.\mathrm{O}_{2}[3], 3\right)\right\}$ (Color figure online)

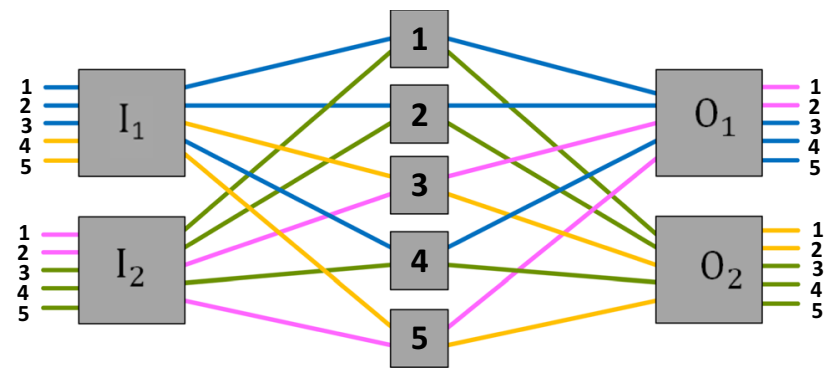

Fig. 2 Three-stage Clos network modeled as the WSW1 switching fabric with the connections routed as shown in Fig. 1 (Color figure online)

$m$-slot connections, where $m$ is limited to two values of $m_{1}$ and $m_{2}$, and $m_{2}>m_{1}$. We also assume that the BV-WSs have full range conversion capability, i.e., an $m$-slot connection that uses a set of $m$ adjacent FSUs at the input fiber can be switched to a set of any other $m$ adjacent FSUs at the output fiber. This conversion from any input to any output FSU is possible only if the converters are working in the full range; otherwise, an algorithm is needed to assign connections to converters depending on the required conversion range such that all of the connections can be switched correctly through the switching fabric. The number of these converters and their range should also be derived. Therefore, these FSUs must be routed by using $m$ adjacent FSUs at the interstage links. Let us consider an $m$-slot connection from input $I_{i}$, which occupies FSUs from $x$ to $x+m-1$, to output switch $O_{j}$ with FSUs assigned from $y$ to $y+m-1$. This connection is denoted by $\left(I_{1}[x], O_{2}[y], m\right)$.

The problem of routing connections in the switching fabric considered can be viewed as similar to routing connections in the three-stage Clos network. The switching fabric presented in Fig. 1 has a set of 2-slot and 3-slot connections for simultaneous setup. The three-stage Clos switching fabric model is presented in Fig. 2. In this model, each input switch in WSW1 is represented by one space switch in the first stage. Each FSU in the input fiber corresponds to one input link in the respective space switch in Fig. 2, and each FSU in the output fiber corresponds to one output link in the respective space switch in Fig. 2. Each FSU in the interstage links corresponds to one space switch in the center stage in Fig. 2. These connections can be represented by the connection matrix $\mathbf{H}=\left[\begin{array}{ll}3 & 2 \\ 2 & 3\end{array}\right]$, which can be decomposed using the Neiman algorithm [20-22] into the following five permutation matrices: $\mathbf{P}_{\mathbf{1}}=\left[\begin{array}{ll}1 & 0 \\ 0 & 1\end{array}\right], \quad \mathbf{P}_{\mathbf{2}}=\left[\begin{array}{ll}1 & 0 \\ 0 & 1\end{array}\right], \quad \mathbf{P}_{\mathbf{3}}=\left[\begin{array}{ll}0 & 1 \\ 1 & 0\end{array}\right]$, $\mathbf{P}_{4}=\left[\begin{array}{ll}1 & 0 \\ 0 & 1\end{array}\right]$, and $\mathbf{P}_{5}=\left[\begin{array}{ll}0 & 1 \\ 1 & 0\end{array}\right]$. Each permutation matrix represents the connections routed through one switch in the middle stage. The FSUs belonging to connections represented by $\mathbf{P}_{\mathbf{1}}$ are set up through the first switch (or first FSUs in interstage links), for $\mathbf{P}_{\mathbf{2}}$ through the second switch, $\mathbf{P}_{\mathbf{3}}$ through the third switch, and similarly for $\mathbf{P}_{\mathbf{4}}$ and $\mathbf{P}_{\mathbf{5}}$. As a result, the first connection $\left(I_{1}[1], O_{1}[3], 3\right)$ (marked in blue in Fig. 1) is set up through FSUs 1, 2, and 4 in the interstage link in Fig. 2, which is not correct because these FSUs are not adjacent, but this is required according to [4]. The same problem occurs with the connection $\left(I_{1}[4], O_{2}[1], 2\right)$ (marked in orange in Figs. 1 and 2), which is set up through FSUs 3 and 5, and connection $\left(I_{2}[3], O_{2}[3], 3\right)$ (marked in green in Figs. 1 and 2) with the assigned FSUs 1, 2, and 4. This example shows that the problem of routing simultaneous connections in WSW1 appears to be similar to routing in the three-stage Clos network, but there are important differences, as follows:

(i) Instead of finding connections that can be set up through one center stage switch, we must find connections that can be set up using FSUs with the same sequence numbers in the interstage links.

(ii) Connections can occupy FSUs with different sequence numbers, but the FSUs occupied by each connection must be adjacent.

The first problem is caused by the lack of conversion capabilities in the center-stage switch. Therefore, connection $\left(I_{1}\left[x_{1}\right], O_{2}\left[y_{1}\right], m\right)$ can be set up through $m$ FSUs by starting from $x_{2}$ in the link from $I_{1}$ and by starting from $y_{2}$ in the link to $O_{2}$ only if $x_{2}=y_{2}$. The second problem is related to the standard [4] and the manner in which modulation formats occupy the bandwidth. For these reasons, the algorithms and solutions used for Clos networks cannot be used directly in the switching fabric considered. In the present study, we consider the simultaneous connection model. It is assumed that we have a set of compatible connection requests, i.e., connection requests occupy as many FSUs as possible in each input and output fibers. Thus, in such set, no more $m_{2}$ -slot and $m_{1}$-slot connection requests can be added.

The set of compatible connections is denoted by $\mathbb{C}$ and an example of this type of set in a $2 \times 2$ switching fabric 


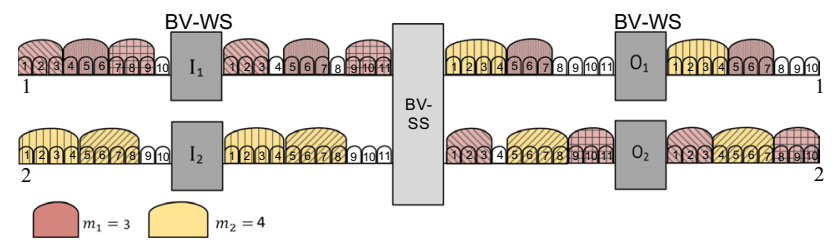

Fig. 3 WSW1 switching fabric with a set of connection requests, $\mathbb{C}=\left\{\left(I_{1}[1], O_{2}[1], 3\right) ;\left(I_{1}[4], O_{2}[8], 3\right) ;\left(I_{1}[7], O_{1}[5], 3\right) ;\left(I_{2}[1]\right.\right.$, $\left.\left.O_{1}[1], 4\right) ;\left(I_{2}[5], O_{2}[4], 4\right)\right\}$, set up through 11 FSUs

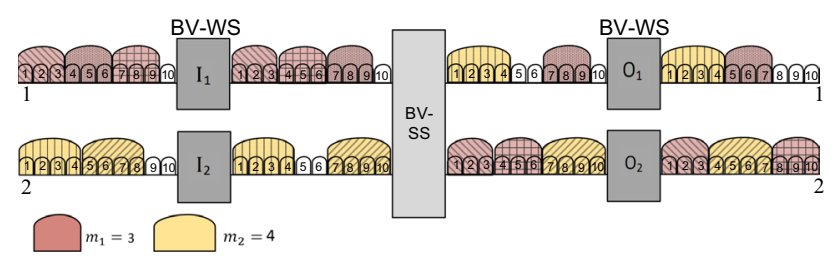

Fig. 4 WSW1 switching fabric with a set of connection requests $\mathbb{C}$ taken from Fig. 3 set up through 10 FSUs

with $n=10$ is shown in Fig. 3. Five connections of two types need to be set up comprising two 4-slot connections, and three 3 -slot connections. Therefore, the problem is determining which FSUs in the interstage links should be used by these connections and how many FSUs are needed to set up all possible sets of compatible connections, i.e., when the switching fabric is RNB. For instance, connections $\left(I_{1}[1], O_{2}[1], 3\right)$ and $\left(I_{2}[1], O_{1}[1], 4\right)$ are directed from different input switches to different output switches, so they are not in conflict in the BV-SS and they can be set up using the same FSUs numbered from 1 to 4 in the interstage links (FSU number 4 will remain free in the first interstage link). When all of the connections are set up, the number of FSUs required in the interstage links is 11. However, this set of connections can be set up in a different way, as shown in Fig. 4. In this case, connections $\left(I_{1}[1], O_{2}[1], 3\right)$ and $\left(I_{1}[4], O_{2}[8], 3\right)$ are set up using FSUs with the same sequence numbers as connection $\left(I_{2}[1], O_{1}[1], 4\right)$, and the number of FSUs required in the interstage links is 10 . Both approaches are considered in Sects. 4 and 5, where the RNB conditions are derived for these algorithms.

\section{Model description}

In this study, we use the model proposed by [19], but we focus only on the $2 \times 2$ switching fabric, where we do not impose any limits on the ratio between $n, m_{1}$, and $m_{2}$. Let $\mathbb{C}$ be set up in the WSW1 switching fabric, and it contains only connections with two rates: $m_{1}$ and $m_{2}$, where $m_{1}<m_{2}$. For instance, in the set of connection requests presented in Fig. 3, $m_{1}=3$ and $m_{2}=4$. We use two connection matrices denoted by $\mathbf{H}^{m_{1}}$ and $\mathbf{H}^{m_{2}}$ to represent the connection requests and each matrix represents the connection requests for one connection rate. The matrix $\mathbf{H}^{\boldsymbol{m}_{x}}$ represents $m_{x}$-slot connections, where $x=1,2$, and it is defined as follows:

$\mathbf{H}^{\boldsymbol{m}_{x}}=\left[\begin{array}{ll}h_{11}^{m_{x}} & h_{12}^{m_{x}} \\ h_{21}^{m_{x}} & h_{22}^{n_{x}}\end{array}\right]$,

where $h_{i j}^{m_{x}}$ is equal to the number of $m_{x}$-slot connection requests from switch $I_{i}$ to switch $O_{j}$. For instance, the connection matrices for the set of connection requests in Fig. 3 are as follows:

$\mathbf{H}^{m_{1}}=\left[\begin{array}{ll}1 & 2 \\ 0 & 0\end{array}\right], \quad \mathbf{H}^{m_{2}}=\left[\begin{array}{ll}0 & 0 \\ 1 & 1\end{array}\right]$.

The set of matrices $\mathbf{H}^{\boldsymbol{m}_{x}}$ has the following properties:

- for each row $i$,

$$
\sum_{j=1}^{2}\left\{\sum_{x=1}^{2}\left(h_{i j}^{m_{x}} \cdot m_{x}\right)\right\} \leqslant n,
$$

- and for each column $j$,

$$
\sum_{i=1}^{2}\left\{\sum_{x=1}^{2}\left(h_{i j}^{m_{x}} \cdot m_{x}\right)\right\} \leqslant n .
$$

Formula (3) states that the sum of FSUs used by all connection requests from one input fiber is not greater than $n$. When $\left\lfloor\frac{n}{m_{2}}\right\rfloor$ and $\left\lfloor\frac{n}{m_{1}}\right\rfloor$ are not integers, the number of occupied FSUs may be less than $n$. Formula (4) provides the same condition for the output fibers, where it states that the number of FSUs used by the connection requests to one output fiber must also be no greater than $n$.

We also use the following terms and notation:

- $a_{i}^{m_{x}}$ denotes the number of $m_{x}$-slot connection requests at input $i: a_{i}^{m_{x}}=\sum_{j=1}^{2} h_{i j}^{m_{x}}$;

- $b_{j}^{m_{x}}$ denotes the number of $m_{x}$-slot connection requests at output $j: b_{j}^{m_{x}}=\sum_{i=1}^{2} h_{i j}^{m_{x}}$;

- $a_{\text {max }}^{m_{x}}$ denotes the maximum number of $m_{x}$-slot connection requests at one input: $a_{\max }^{m_{x}}=\max _{1 \leqslant i \leqslant 2}\left\{a_{i}^{m_{x}}\right\}$;

- $a_{\text {min }}^{m_{x}}$ denotes the minimum number of $m_{x}$-slot connection requests at one input: $a_{\min }^{m_{x}}=\min _{1 \leqslant i \leqslant 2}\left\{a_{i}^{m_{x}}\right\}$;

- $b_{\max }^{m_{x}}$ denotes the maximum number of $m_{x}$-slot connection requests at one output: $b_{\max }^{m_{x}}=\max _{1 \leqslant j \leqslant 2}\left\{b_{j}^{m_{x}}\right\}$;

- $b_{\text {min }}^{m_{x}}$ denotes the minimum number of $m_{x}$-slot connection requests at one output: $b_{\text {min }}^{m_{x}}=\min _{1 \leqslant j \leqslant 2}\left\{b_{j}^{m_{x}}\right\}$;

- $c_{\max }^{m_{x}}=\max \left\{a_{\max }^{m_{x}} ; b_{\max }^{m_{x}}\right\}$;

$-c_{\min }^{m_{x}}=\min \left\{a_{\min }^{m_{x}} ; b_{\min }^{m_{x}}\right\}$. 


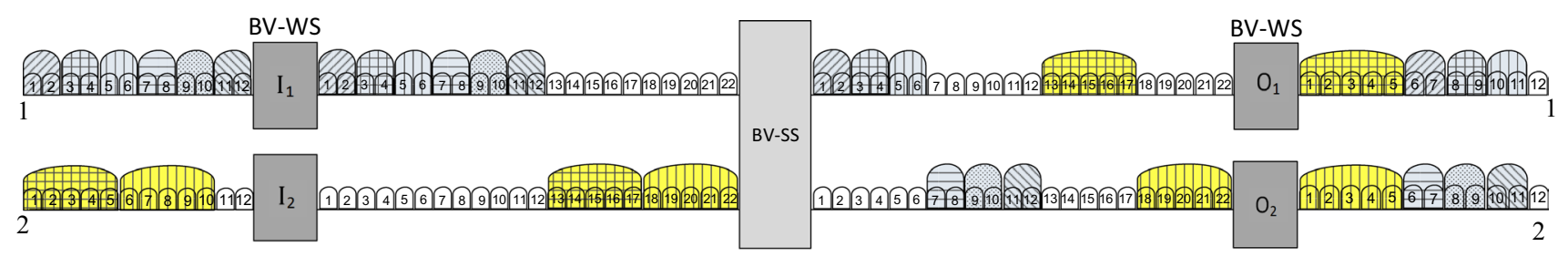

$m_{1}=2$

$m_{2}=5$

Fig. 5 The $2 \times 2$ WSW1 switching fabric with a set of connection requests, $\mathbb{C}=\left\{\left(I_{1}[1], O_{1}[6], 2\right) ;\left(I_{1}[3], O_{1}[8], 2\right) ;\left(I_{1}[5], O_{1}[10], 2\right)\right.$; $\left.\left(I_{1}[7], O_{2}[6], 2\right) ;\left(I_{1}[9], O_{2}[8], 2\right) ;\left(I_{1}[11], O_{2}[10], 2\right) ;\left(I_{2}[1], O_{1}[1], 5\right) ;\left(I_{2}[6], O_{2}[1], 5\right)\right\}$

\section{Control algorithm}

The problem of assigning FSUs to a particular connection request can be solved using the matrix decomposition algorithm. It is known that the square matrix $\mathbf{H}$ can be decomposed into $n$ permutation matrices when the sum of the elements in each row and each column is equal to $n[13,16]$. However, this is not true in our case, so the decomposition algorithm must be modified.

We have a set of compatible connection requests $\mathbb{C}$. For each connection rate, $\mathbf{H}^{\boldsymbol{m}_{\boldsymbol{x}}}$ is calculated in the first step. In the next step, $a_{i}^{m_{x}}$ and $b_{j}^{m_{x}}$ are calculated for each row and column, respectively. If these values are equal, $\mathbf{H}^{\boldsymbol{m}_{x}}$ can be decomposed into permutation matrices. If these numbers are not equal, "dummy" connection requests are added such that these sums will be equal to $c_{\max }^{m_{x}}=\max \left\{a^{m_{x}} ; b^{m_{x}}\right\}$. In fact, "dummy" connection requests are not present in the set of connections, but instead, they employ resources that are not used by the other connection requests represented in $\mathbf{H}^{\boldsymbol{m}_{\boldsymbol{x}}}$, and they are only added to ensure the appropriate operation of the decomposition algorithm, and they are not set up in the switching fabric at the end.

Matrix $\mathbf{H}^{\boldsymbol{m}_{\boldsymbol{x}}}$ is then decomposed into $c_{\max }^{m_{x}}$ permutation matrices $\mathbf{P}_{i}^{\boldsymbol{m}_{\boldsymbol{x}}}, 1 \leqslant i \leqslant c_{\max }^{m_{x}}$, using any known algorithm [20-22]. One matrix $\mathbf{P}_{i}^{m_{x}}$ represents a set of connections that can be set up through the interstage links using $m_{x}$ FSUs with the same sequence numbers. Each entry $p_{i j}=1$ denotes one connection request $\left(I_{i}[x], O_{j}[y], m_{x}\right)$. Finally, some permutation matrices elements representing "dummy" connection requests must be removed. As the result, some rows and columns in the permutation matrices will only contain " 0 " elements, and these matrices are called partial permutation matrices. For instance, in the following partial permutation matrix:

$\left[\begin{array}{llll}0 & 0 & 0 & 0 \\ 0 & 1 & 0 & 0 \\ 0 & 0 & 0 & 0 \\ 0 & 0 & 1 & 0\end{array}\right]$ rows 1 and 3, and columns 1 and 4 do not contain any " 1 " elements. Therefore, inputs 1 and 3, and outputs 1 and 4 remain unassigned in this matrix. The decomposition algorithm is presented in Algorithm 1.

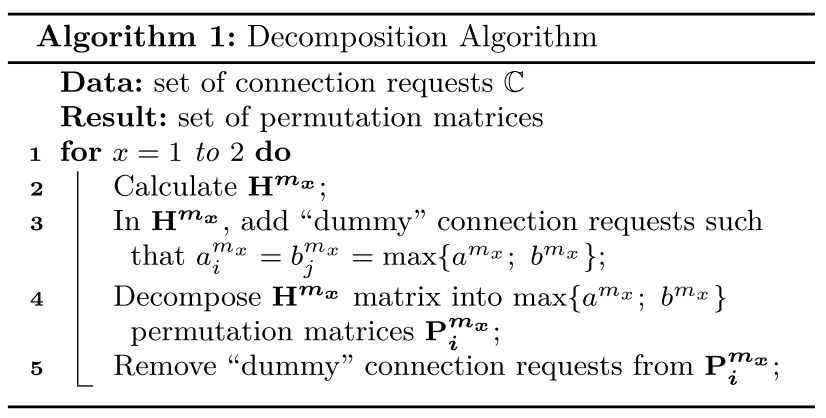

In our case, from $c_{\max }^{m_{x}}$ matrices $\mathbf{P}_{i}^{m_{x}}, c_{\min }^{m_{x}}$ matrices are full permutation matrices (where every row and every column contains exactly one nonzero entry of " 1 "), whereas $\left(c_{\max }^{m_{x}}-c_{\min }^{m_{x}}\right)$ matrices are partial permutation matrices.

The example in Fig. 5 illustrates how the algorithm works. We have $n=12$ FSUs in each input/output link. The switching fabric serves eight connections with sizes of $m_{1}=2$ and $m_{2}=5$. In the second input, we have two free FSUs, but they cannot be used by an additional 2-slot connection because we only have one free FSU in each output link. In this example, matrices $\mathbf{H}^{\boldsymbol{m}_{x}}$ are:

$\mathbf{H}^{\boldsymbol{m}_{1}}=\left[\begin{array}{ll}3 & 3 \\ 0 & 0\end{array}\right], \quad \mathbf{H}^{\boldsymbol{m}_{2}}=\left[\begin{array}{ll}0 & 0 \\ 1 & 1\end{array}\right]$.

We have $a_{1}^{m_{1}}=6, a_{2}^{m_{1}}=0$, and $b_{1}^{m_{1}}=b_{2}^{m_{1}}=3$. In order to decompose this matrix, we have added six "dummy" connection requests in positions $h_{21}^{m_{1}}$ and $h_{22}^{m_{1}}$ (three of these connection requests for each position, where the connections are marked by gray circles), so we obtain:

$\mathbf{H}^{\boldsymbol{m}_{\mathbf{1}}}=\left[\begin{array}{ll}3 & 3 \\ 3 & 3\end{array}\right]$. 
Table 1 Assignment of FSUs for connection requests $\mathbb{C}$ in Fig. 5

\begin{tabular}{lll}
\hline Permutation matrix & Connection & Assigned FSUs \\
\hline $\mathbf{P}_{\mathbf{m}}^{\boldsymbol{m}_{1}}$ & $\left(I_{1}[1], O_{1}[6], 2\right)$ & $1-2$ \\
$\mathbf{P}_{\mathbf{2}}^{\boldsymbol{m}_{1}}$ & $\left(I_{1}[3], O_{1}[8], 2\right)$ & $3-4$ \\
$\mathbf{P}_{\mathbf{3}}^{\boldsymbol{m}_{1}}$ & $\left(I_{1}[5], O_{1}[10], 2\right)$ & $5-6$ \\
$\mathbf{P}_{\mathbf{4}}^{\boldsymbol{m}_{1}}$ & $\left(I_{1}[7], O_{2}[6], 2\right)$ & $7-8$ \\
$\mathbf{P}_{\mathbf{5}}^{\boldsymbol{m}_{1}}$ & $\left(I_{1}[9], O_{2}[8], 2\right)$ & $9-10$ \\
$\mathbf{P}_{\mathbf{6}}^{\boldsymbol{m}_{1}}$ & $\left(I_{1}[11], O_{2}[10], 2\right)$ & $11-12$ \\
$\mathbf{P}_{\mathbf{1}}^{\boldsymbol{m}_{\mathbf{2}}}$ & $\left(I_{2}[1], O_{1}[1], 5\right)$ & $13-17$ \\
$\mathbf{P}_{\mathbf{2}}^{\boldsymbol{m}_{\mathbf{2}}}$ & $\left(I_{2}[6], O_{2}[1], 5\right)$ & $18-22$ \\
\hline
\end{tabular}

This matrix can be decomposed into six permutation matrices, which are partial permutation matrices after removing the "dummy" connection requests:

$\mathbf{P}_{\mathbf{1}}^{m_{1}}=\mathbf{P}_{\mathbf{2}}^{m_{1}}=\mathbf{P}_{\mathbf{3}}^{\boldsymbol{m}_{1}}=\left[\begin{array}{ll}1 & 0 \\ 0 & 1\end{array}\right] \Longrightarrow\left[\begin{array}{ll}1 & 0 \\ 0 & 0\end{array}\right]$,

$\mathbf{P}_{4}^{m_{1}}=\mathbf{P}_{5}^{m_{1}}=\mathbf{P}_{6}^{m_{1}}=\left[\begin{array}{ll}0 & 1 \\ 1 & 0\end{array}\right] \Longrightarrow\left[\begin{array}{ll}0 & 1 \\ 0 & 0\end{array}\right]$.

These connection requests in the interstage links are implemented through FSUs with the sequence numbers shown in Table 1. Partial permutation matrix $\mathbf{P}_{1}^{m_{1}}$ represents 2-slot connection $\left(I_{1}[1], O_{1}[6], 2\right)$, and it is set up using FSUs 1 and 2 in the interstage links, and $\mathbf{P}_{2}^{m_{1}}$ represents 2 -slot connection $\left(I_{1}[3], O_{1}[8], 2\right)$ and it uses FSUs 3 and 4, and so on.

For $\mathbf{H}^{\boldsymbol{m}_{2}}$ we have: $a_{1}^{m_{2}}=0, a_{2}^{m_{2}}=2$, and $b_{1}^{m_{2}}=b_{2}^{m_{2}}=1$. In order to decompose this matrix, we have added two "dummy" connection requests in positions $h_{11}^{m_{2}}$ and $h_{12}^{m_{2}}$ (positions with added connection requests are marked by gray circles), so we obtain:

$\mathbf{H}^{\boldsymbol{m}_{\mathbf{2}}}=\left[\begin{array}{cc}1 & 1 \\ 1 & 1\end{array}\right]$

This matrix can be decomposed into two permutation matrices, which are partial permutation matrices after removing the "dummy" connection requests:

$\mathbf{P}_{1}^{m_{2}}=\left[\begin{array}{ll}0 & 1 \\ 1 & 0\end{array}\right] \Longrightarrow\left[\begin{array}{ll}0 & 0 \\ 1 & 0\end{array}\right]$
$\mathbf{P}_{\mathbf{2}}^{\boldsymbol{m}_{\mathbf{2}}}=\left[\begin{array}{ll}1 & 0 \\ 0 & 1\end{array}\right] \Longrightarrow\left[\begin{array}{ll}0 & 0 \\ 0 & 1\end{array}\right]$

The connections corresponding to $\mathbf{P}_{1}^{m_{2}}$ and $\mathbf{P}_{2}^{m_{2}}$, and the numbers of assigned FSUs are also given in Table 1.

In the example considered, the number of FSUs required in the interstage links is $22(k=22)$ because Algorithm 1 assumes that each set of $m_{x}$-slot connections is set up through FSUs with different sequence numbers in the interstage links. However, the number of FSUs required can be reduced when some of the FSUs assigned for connection requests with size $m_{2}$ can also be used by connection requests with size $m_{1}$, or vice versa. For instance, in the example of Fig. 5, connection $\left(I_{2}[1], O_{1}[1], 5\right)$ represented by $\mathbf{P}_{1}^{m_{2}}$ can use the same FSUs in the interstage links as connections $\left(I_{1}[7], O_{2}[6], 2\right)$ and $\left(I_{1}[9], O_{2}[8], 2\right)$ represented by $\mathbf{P}_{4}^{m_{1}}$ and $\mathbf{P}_{5}^{m_{1}}$, respectively, because the " 1 " elements in $\mathbf{P}_{1}^{m_{2}}$ and $\mathbf{P}_{\mathbf{4}}^{m_{1}}$ (and in $\mathbf{P}_{1}^{m_{2}}$ and $\mathbf{P}_{5}^{m_{1}}$ as well) are in different rows and columns:

$\mathbf{P}_{1}^{m_{2}}=\left[\begin{array}{ll}0 & 0 \\ 1 & 0\end{array}\right], \quad \mathbf{P}_{4}^{m_{1}}=\mathbf{P}_{5}^{m_{1}}=\left[\begin{array}{ll}0 & 1 \\ 0 & 0\end{array}\right]$.

Thus, we can say that matrices $\mathbf{P}_{1}^{m_{2}}$ and $\mathbf{P}_{\mathbf{4}}^{m_{1}}\left(\right.$ and $\mathbf{P}_{1}^{m_{2}}$ and $\mathbf{P}_{5}^{m_{1}}$ ) can be merged together. In general, matrices $\mathbf{P}_{i}^{m_{2}}$ and $\mathbf{P}_{j}^{m_{1}}$ can be merged together when the result matrix is also a permutation (or a partial permutation) matrix. Matrices can be merged in two different ways:

(i) Case 1 One matrix $\mathbf{P}^{\boldsymbol{m}_{2}}$ can be merged with $\left\lfloor\frac{m_{2}}{m_{1}}\right\rfloor$ matrices $\mathbf{P}^{m_{1}}$,

(ii) Case $2\left\lceil\frac{m_{2}}{m_{1}}\right\rceil$ matrices $\mathbf{P}^{m_{1}}$ can be merged with one $\operatorname{matrix} \mathbf{P}^{m_{2}}$.

The algorithm is presented as Algorithm 2 with two options for $t_{1}=\left\lfloor\frac{m_{2}}{m_{1}}\right\rfloor$ and $t_{2}=\left\lceil\frac{m_{2}}{m_{1}}\right\rceil$. The first option is denoted as MA1. It starts by checking the partial permutation matrix $\mathbf{P}_{i}^{m_{2}}$ to determine which position element " 1 " is present. When this element is in row $v$ and column $w$ $\left(h_{v w}^{m_{2}}=1\right)$, the algorithm looks for $\mathbf{P}_{i}^{\boldsymbol{m}_{1}}$ with $a_{v}^{m_{1}}=0$ and $b_{w}^{m_{1}}=0$. Up to $\left\lfloor\frac{m_{2}}{m_{1}}\right\rfloor \mathbf{P}_{\boldsymbol{i}}^{m_{1}}$, matrices can be merged with one $\mathbf{P}_{i}^{m_{2}}$ matrix. This operation is repeated for each partial permutation matrix $\mathbf{P}_{i}^{m_{2}}$. 


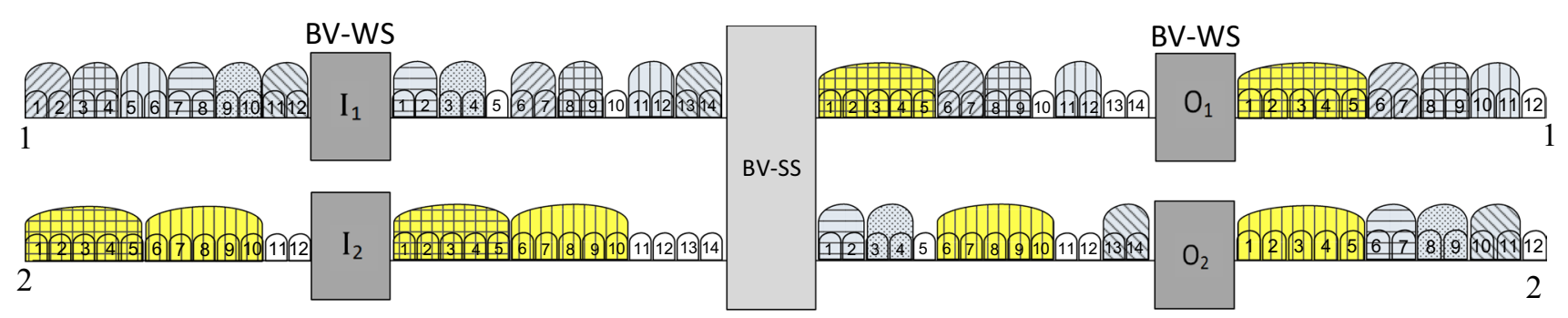

$m_{1}=2$

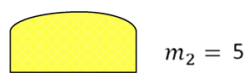

Fig. 6 The $2 \times 2$ WSW1 switching fabric shown in Fig. 5 with $\mathbb{C}$ set through 14 FSUs according to MA1

Table 2 Assignment of FSUs for connection requests $\mathbb{C}$ according to MA1

\begin{tabular}{|c|c|c|c|c|}
\hline $\begin{array}{l}\text { Permutation } \\
\text { matrix }\end{array}$ & $\begin{array}{l}\text { Merged permuta- } \\
\text { tion matrix }\end{array}$ & Connection & Merged connection & Assigned FSUs \\
\hline $\mathbf{P}_{1}^{m_{2}}$ & - & $\left(I_{2}[1], O_{1}[1], 5\right)$ & - & $1-5$ \\
\hline- & $\mathbf{P}_{4}^{m_{1}}$ & - & $\left(I_{1}[7], O_{2}[6], 2\right)$ & $1-2$ \\
\hline- & $\mathbf{P}_{5}^{m_{1}}$ & - & $\left(I_{1}[9], O_{2}[8], 2\right)$ & $3-4$ \\
\hline $\mathbf{P}_{2}^{m_{2}}$ & - & $\left(I_{2}[6], O_{2}[1], 5\right)$ & - & $6-10$ \\
\hline- & $\mathbf{P}_{1}^{m_{1}}$ & - & $\left(I_{1}[1], O_{1}[6], 2\right)$ & $6-7$ \\
\hline- & $\mathbf{P}_{2}^{m_{1}}$ & - & $\left(I_{1}[3], O_{1}[8], 2\right)$ & $8-9$ \\
\hline $\mathbf{P}_{3}^{m_{1}}$ & - & $\left(I_{1}[5], O_{1}[10], 2\right)$ & - & $11-12$ \\
\hline $\mathbf{P}_{6}^{m_{1}}$ & - & $\left(I_{1}[11], O_{2}[10], 2\right)$ & - & $13-14$ \\
\hline
\end{tabular}

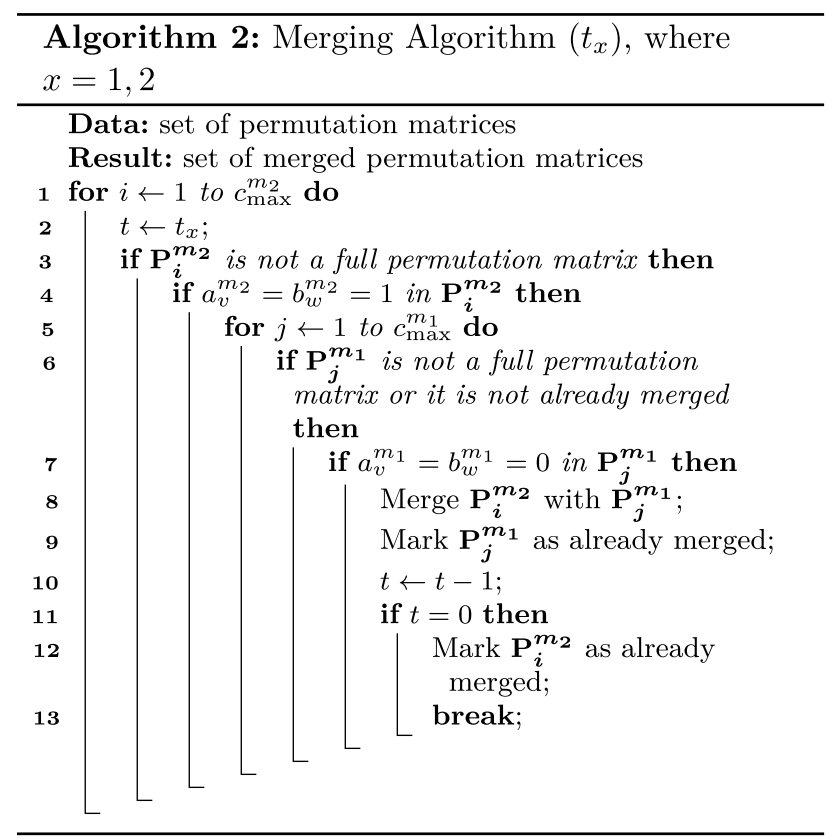

The second option (Case 2) is denoted as MA2 and we merge $\left\lceil\frac{m_{2}}{m_{1}}\right\rceil$ matrices $\mathbf{P}_{j}^{m_{1}}$ with one matrix $\mathbf{P}_{i}^{\boldsymbol{m}_{2}}$. The algorithm starts by finding element " 1 " in partial permutation matrix $\mathbf{P}_{i}^{m_{2}}$. When $h_{v w}^{m_{2}}=1$, MA2 searches for $\mathbf{P}_{j}^{m_{1}}$ where $a_{v}^{m_{1}}=0$ and $b_{w}^{m_{1}}=0$. If this matrix is present, $\mathbf{P}_{\boldsymbol{i}}^{\boldsymbol{m}_{\mathbf{2}}}$ and $\mathbf{P}_{\boldsymbol{j}}^{\boldsymbol{m}_{1}}$ can be merged together. Then, we check whether the next $\mathbf{P}_{j}^{m_{1}}$ can be merged with $\mathbf{P}_{i}^{m_{2}}$. The merging process is finished when we have found $\left\lceil\frac{m_{2}}{m_{1}}\right\rceil$ matrices $\mathbf{P}_{j}^{m_{1}}$ or there are no more $\mathbf{P}_{i}^{m_{2}}$ or $\mathbf{P}_{j}^{m_{1}}$ matrices.

Let us again consider the example shown in Fig. 5. When MA1 is used, partial permutation matrix $\mathbf{P}_{\mathbf{1}}^{\mathbf{m}_{2}}$ can be merged with matrices $\mathbf{P}_{4}^{m_{1}}$ and $\mathbf{P}_{5}^{m_{1}}$, and the connection requests corresponding to these matrices use FSUs from 1 to 5. The next partial permutation matrix $\mathbf{P}_{\mathbf{2}}^{\boldsymbol{m}_{2}}$ can be merged with matrices $\mathbf{P}_{1}^{m_{1}}$ and $\mathbf{P}_{2}^{m_{1}}$, and the connection requests corresponding to these matrices use FSUs from 6 to 10. Matrices $\mathbf{P}_{\mathbf{3}}^{m_{1}}$ and $\mathbf{P}_{\mathbf{6}}^{m_{1}}$ are not merged and their connection requests use FSUs from 11 to 14. Figure 6 shows the state of the switching fabric, and Table 2 shows the merged permutation matrices and FSUs assigned to connection requests $\mathbb{C}$.

When MA2 is used, the results obtained by the merging operations are shown in Table 3 and Fig. 7. In this case, $\left\lceil\frac{m_{2}}{m_{1}}\right\rceil=3$ and matrices $\mathbf{P}_{\mathbf{4}}^{m_{1}}, \mathbf{P}_{\mathbf{5}}^{\boldsymbol{m}_{\mathbf{1}}}$, and $\mathbf{P}_{\mathbf{6}}^{\boldsymbol{m}_{\mathbf{1}}}$ can be merged with matrix $\mathbf{P}_{1}^{m_{2}}$, where the respective connection requests use FSUs from 1 to 6 . In the same manner, matrices $\mathbf{P}_{1}^{m_{1}}$, $\mathbf{P}_{2}^{m_{1}}$, and $\mathbf{P}_{3}^{m_{1}}$ can be merged with matrix $\mathbf{P}_{2}^{m_{2}}$, and their connection requests use FSUs from 7 to 12 .

Therefore, the number of FSUs required is reduced from 22 to 14 when using MA1, whereas MA2 reduces this number to 12 . The numbers of FSUs required with 


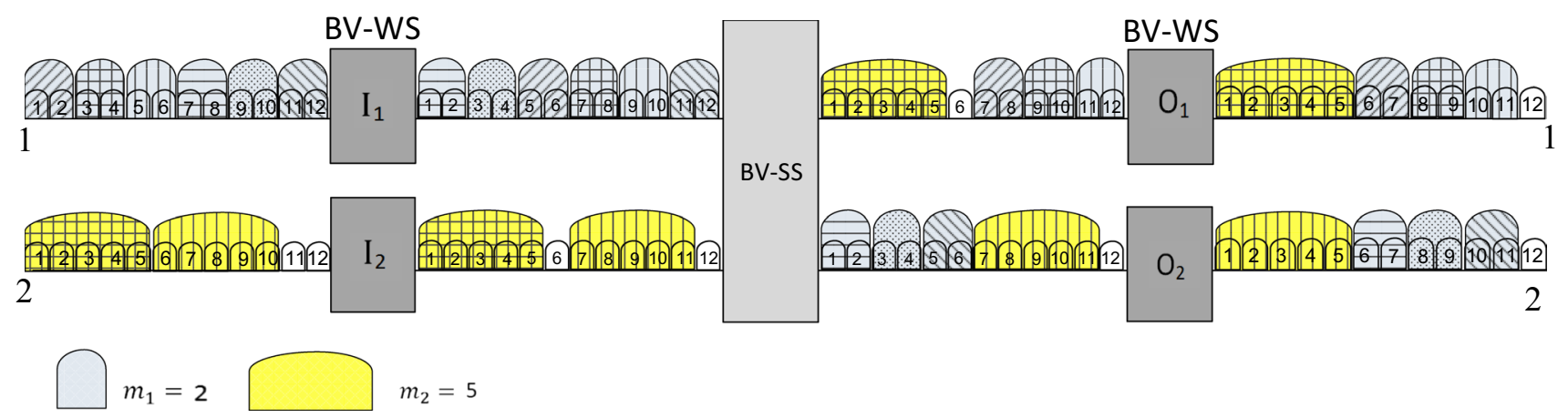

Fig. 7 The $2 \times 2$ WSW1 switching fabric shown in Fig. 5 with $\mathbb{C}$ set through 12 FSUs according to MA2

Table 3 Assignment of FSUs for connection requests $\mathbb{C}$ according to MA2

\begin{tabular}{lllll}
\hline $\begin{array}{l}\text { Permutation } \\
\text { matrix }\end{array}$ & $\begin{array}{l}\text { Merged permuta- } \\
\text { tion matrix }\end{array}$ & Connection & Merged connection & Assigned FSUs \\
\hline $\mathbf{P}_{\mathbf{m}}^{m_{1}}$ & - & $\left(I_{1}[7], O_{2}[6], 2\right)$ & - & $1-2$ \\
$\mathbf{P}_{\mathbf{5}}^{m_{1}}$ & - & $\left(I_{1}[9], O_{2}[8], 2\right)$ & - & $3-4$ \\
$\mathbf{P}_{\mathbf{6}}^{m_{1}}$ & - & $\left(I_{1}[11], O_{2}[10], 2\right)$ & - & $5-6$ \\
- & $\mathbf{P}_{\mathbf{1}}^{\boldsymbol{m}_{\mathbf{2}}}$ & - & $\left(I_{2}[1], O_{1}[1], 5\right)$ & $1-5$ \\
$\mathbf{P}_{\mathbf{1}}^{\boldsymbol{m}_{\mathbf{1}}}$ & - & $\left(I_{1}[1], O_{1}[6], 2\right)$ & - & $7-8$ \\
$\mathbf{P}_{\mathbf{2}}^{m_{1}}$ & - & $\left(I_{1}[3], O_{1}[8], 2\right)$ & - & $9-10$ \\
$\mathbf{P}_{\mathbf{3}}^{m_{1}}$ & - & $\left(I_{1}[5], O_{1}[10], 2\right)$ & - & $11-12$ \\
- & - & - & $\left(I_{2}[6], O_{2}[1], 5\right)$ & $7-11$ \\
\hline
\end{tabular}

both merging algorithms are considered in the two theorems presented in the next section.

\section{Rearrangeable conditions}

Next, we need to determine how many FSUs are needed in the interstage links to implement all possible sets of compatible connections using the decomposition algorithm together with one of the proposed merging algorithms.

Theorem 1 The $2 \times 2$ WSW1 switching fabric is RNB for $m$-slot connections, where $m \in\left\{m_{1} ; m_{2}\right\}$ under MAl if:

$k_{\mathrm{MA} 1}^{2} \geqslant\left\lfloor\frac{n}{m_{2}}\right\rfloor \cdot m_{2}+\left(\left\lfloor\frac{n}{m_{1}}\right\rfloor-\left\lfloor\frac{n}{m_{2}}\right\rfloor \cdot\left\lfloor\frac{m_{2}}{m_{1}}\right\rfloor\right) \cdot m_{1}$.

Proof Let $\mathbb{C}$ denote a set of compatible connection requests. We have two connection rates, $m_{1}$ and $m_{2}$, and all of the connection requests in $\mathbb{C}$ are represented by matrices $\mathbf{H}^{\boldsymbol{m}_{1}}$ and $\mathbf{H}^{\boldsymbol{m}_{2}}$. These matrices can be decomposed into $c_{\max }^{m_{1}}$ and $c_{\max }^{m_{2}}$ permutation matrices $\mathbf{P}^{m_{1}}$ and $\mathbf{P}^{m_{2}}$, respectively. Each $\mathbf{P}^{m_{x}}$ matrix represents a set of $m_{x}$-slot connection requests that can be set up using FSUs with the same sequence numbers in the interstage links. From these $\mathbf{P}^{\boldsymbol{m}_{\boldsymbol{x}}}$, only $c_{\text {min }}^{m_{1}}$ and $c_{\text {min }}^{m_{2}}$ matrices are full permutation matrices. $\left(c_{\max }^{m_{1}}-c_{\min }^{m_{1}}\right)$ for $\mathbf{P}^{m_{1}}$ and $\left(c_{\max }^{m_{2}}-c_{\text {min }}^{m_{2}}\right)$ for $\mathbf{P}^{\boldsymbol{m}_{2}}$ are partial permutation matrices, i.e., at most $\left(c_{\max }^{m_{2}}-c_{\text {min }}^{m_{2}}\right)$ matrices $\mathbf{P}^{m_{2}}$ can be merged with at $\operatorname{most}\left(c_{\max }^{m_{1}}-c_{\min }^{m_{1}}\right)$ matrices $\mathbf{P}^{\boldsymbol{m}_{1}}$.

The number of FSUs required in interstage links $k$ for $\mathbb{C}$ is given by the following formula:

$$
\begin{aligned}
k_{\mathrm{MA} 1}^{2} \geqslant & c_{\min }^{m_{1}} \cdot m_{1}+c_{\min }^{m_{2}} \cdot m_{2}+\left(c_{\max }^{m_{2}}-c_{\min }^{m_{2}}\right) \cdot m_{2} \\
& +\left(\left(c_{\max }^{m_{1}}-c_{\min }^{m_{1}}\right)-\left(c_{\max }^{m_{2}}-c_{\min }^{m_{2}}\right) \cdot\left\lfloor\frac{m_{2}}{m_{1}}\right\rfloor\right) \cdot m_{1} .
\end{aligned}
$$

$c_{\min }^{m_{1}} \cdot m_{1}$ is the number of FSUs used by the connection requests represented in full permutation matrices $\mathbf{P}^{m_{1}}$. We have $c_{\min }^{m_{1}}$ such matrices and each uses $m_{1}$ FSUs. Similarly, there are $c_{\min }^{m_{2}} \mathbf{P}^{m_{2}}$ full permutation matrices and each occupies $m_{2}$ FSUs. Each partial permutation matrix $\mathbf{P}^{\boldsymbol{m}_{2}}$ needs $m_{2}$ FSUs, and there are $\left(c_{\max }^{m_{2}}-c_{\min }^{m_{2}}\right)$ of these matrices. Finally, from $\left(c_{\max }^{m_{1}}-c_{\min }^{m_{1}}\right)$ partial permutation matrices $\mathbf{P}^{m_{1}},\left\lfloor\frac{m_{2}}{m_{1}}\right\rfloor$ of these matrices can be merged with each partial permutation matrix $\mathbf{P}^{m_{2}}$ and each from the remaining $\mathbf{P}^{m_{1}}$ uses $m_{1}$ FSUs.

Formula (14) can be simplified to:

$$
\begin{aligned}
k_{\mathrm{MA} 1}^{2} \geqslant & c_{\max }^{m_{2}} \cdot m_{2} \\
& +\left(c_{\max }^{m_{1}}-\left(c_{\max }^{m_{2}}-c_{\min }^{m_{2}}\right) \cdot\left\lfloor\frac{m_{2}}{m_{1}}\right\rfloor\right) \cdot m_{1} .
\end{aligned}
$$


Formula (15) must be maximized over all possible sets $\mathbb{C}$. $c_{\max }^{m_{x}}$ represents the maximum number of $m_{x}$-slot connection requests in one of the inputs or outputs, so the number of these requests will never be greater than $\left\lfloor\frac{n}{m_{x}}\right\rfloor$. It should be noted that when $c_{\max }^{m_{x}}$ is maximized, $c_{\min }^{m_{x}}$ is minimized, i.e., when $c_{\max }^{m_{2}}=\left\lfloor\frac{n}{m_{2}}\right\rfloor$, which is the maximum number of $m_{2}$-slot connections in one link, the number of $m_{1}$-slot connections in this link is minimized. When we enter $c_{\max }^{m_{1}}=\left\lfloor\frac{n}{m_{1}}\right\rfloor$, $c_{\max }^{m_{2}}=\left\lfloor\frac{n}{m_{2}}\right\rfloor$, and $c_{\text {min }}^{m_{2}}=0$ into formula (15), we obtain the following.

$k_{\mathrm{MA} 1}^{2} \geqslant\left\lfloor\frac{n}{m_{2}}\right\rfloor \cdot m_{2}+\left(\left\lfloor\frac{n}{m_{1}}\right\rfloor-\left\lfloor\frac{n}{m_{2}}\right\rfloor \cdot\left\lfloor\frac{m_{2}}{m_{1}}\right\rfloor\right) \cdot m_{1}$

Theorem 2 The $2 \times 2$ WSW1 switching fabric is RNB for $m$-slot connections, where $m \in\left\{m_{1} ; m_{2}\right\}$ under MA2 if:

$$
\begin{aligned}
k_{\mathrm{MA} 2}^{2} \geqslant\left\lfloor\frac{n-\left\lfloor\frac{n}{m_{2}}\right\rfloor \cdot m_{2}}{m_{1}}\right\rfloor \cdot m_{1}+\left\lfloor\frac{n}{m_{2}}\right\rfloor \cdot m_{2} \\
+\left(\left\lceil\frac{m_{2}}{m_{1}}\right\rfloor \cdot m_{1}-m_{2}\right)\left\lfloor\frac{\left\lfloor\frac{n}{m_{1}}\right\rfloor-\left\lfloor\frac{n-\left\lfloor\frac{n}{m_{2}}\right\rfloor \cdot m_{2}}{m_{1}}\right\rfloor}{\left\lceil\frac{m_{2}}{m_{1}}\right\rfloor}\right\rfloor
\end{aligned}
$$

Proof Similarly as in Theorem 1, matrices $\mathbf{H}^{\boldsymbol{m}_{1}}$ and $\mathbf{H}^{\boldsymbol{m}_{2}}$ representing $\mathbb{C}$ can be decomposed into $c_{\max }^{m_{1}}$ and $c_{\max }^{m_{2}}$ permutation matrices $\mathbf{P}^{m_{1}}$ and $\mathbf{P}^{m_{2}}$, respectively. We have $c_{\min }^{m_{1}}$ and $c_{\min }^{m_{2}}$ full permutation matrices where each uses $m_{1}$ and $m_{2}$ FSUs, respectively. The remaining of matrices $\mathbf{P}^{m_{1}}$ and $\mathbf{P}^{m_{2}}$ are partial permutation matrices. In MA2, we merge $\left\lceil\frac{m_{2}}{m_{1}}\right\rceil$ partial permutation matrices $\mathbf{P}^{m_{1}}$ with one partial permutation matrix $\mathbf{P}^{\boldsymbol{m}_{2}}$. We have $\left(c_{\max }^{m_{2}}-c_{\text {min }}^{m_{2}}\right)$ partial permutation matrices $\mathbf{P}^{m_{2}}$, and when $\left\lceil\frac{m_{2}}{m_{1}}\right\rceil \mathbf{P}^{m_{1}}$ matrices are merged with one $\mathbf{P}^{m_{2}}$, we have $\left(\left\lceil\frac{m_{2}}{m_{1}}\right\rceil \cdot m_{1}-m_{2}\right)$ free FSUs between two successive $m_{2}$-slot connections. We have $\left(c_{\max }^{m_{1}}-c_{\min }^{m_{1}}\right)$ partial permutation matrices $\mathbf{P}^{m_{1}}$ so we can merge them with up to $\left[\frac{c_{\max }^{m_{1}}-c_{\min }^{m_{1}}}{\left\lceil\frac{m_{2}}{m_{1}}\right\rceil}\right] \mathbf{P}^{\boldsymbol{m}_{2}}$ permutation matrices. In this case, the number of FSUs required in interstage links $k$ is given by the following formula:

$$
\begin{aligned}
k_{\mathrm{MA} 2}^{2} \geqslant & c_{\min }^{m_{1}} \cdot m_{1}+c_{\min }^{m_{2}} \cdot m_{2}+\left(c_{\max }^{m_{2}}-c_{\min }^{m_{2}}\right) \cdot m_{2} \\
& +\left(\left\lceil\frac{m_{2}}{m_{1}}\right\rceil \cdot m_{1}-m_{2}\right) \cdot\left\lfloor\frac{c_{\max }^{m_{1}}-c_{\min }^{m_{1}}}{\left\lceil\frac{m_{2}}{m_{1}}\right\rceil}\right\rfloor .
\end{aligned}
$$

Inequality (18) can be simplified to:

$$
\begin{aligned}
k_{\mathrm{MA} 2}^{2} \geqslant & c_{\min }^{m_{1}} \cdot m_{1}+c_{\max }^{m_{2}} \cdot m_{2} \\
& +\left(\left\lceil\frac{m_{2}}{m_{1}}\right\rceil \cdot m_{1}-m_{2}\right) \cdot\left\lfloor\frac{c_{\max }^{m_{1}}-c_{\min }^{m_{1}}}{\left\lceil\frac{m_{2}}{m_{1}}\right\rceil}\right\rfloor
\end{aligned}
$$

Inequality (19) must be maximized through all possible sets $\mathbb{C}$ and it reaches the maximum when $c_{\max }^{m_{2}}$ is maximized, $c_{\max }^{m_{1}}$ is maximized, and $c_{\min }^{m_{1}}$ is also maximized (since $m_{1} \geqslant\left\lceil\frac{m_{2}}{m_{1}}\right\rceil \cdot m_{1}-m_{2}$ ). The respective maximum values are:

$-c_{\max }^{m_{2}}=\left\lfloor\frac{n}{m_{2}}\right\rfloor$, i.e., all connections in one link are $m_{2}$-slot connections;

$-c_{\max }^{m_{1}}=\left\lfloor\frac{n}{m_{1}}\right\rfloor$, i.e., all connections in one link are $m_{1}$-slot connections;

$-c_{\min }^{m_{1}}=\left\lfloor\frac{n-\left\lfloor\frac{n}{m_{2}}\right\rfloor \cdot m_{2}}{m_{1}}\right\rfloor$, i.e., the number of $m_{1}$-slot connections that can be implemented in the link already serving $\left\lfloor\frac{n}{m_{2}}\right\rfloor m_{2}$-slot connections.

By entering these values in formula (19), we obtain the following.

$$
\begin{aligned}
& k_{\mathrm{MA} 2}^{2} \geqslant\left\lfloor\frac{n-\left\lfloor\frac{n}{m_{2}}\right\rfloor \cdot m_{2}}{m_{1}}\right\rfloor \cdot m_{1}+\left\lfloor\frac{n}{m_{2}}\right\rfloor \cdot m_{2} \\
&+\left(\left\lfloor\frac{m_{2}}{m_{1}}\right\rfloor \cdot m_{1}-m_{2}\right)\left\lfloor\frac{\left\lfloor\frac{n}{m_{1}}\right\rfloor-\left\lfloor\frac{n-\left\lfloor\frac{n}{m_{2}}\right\rfloor m_{2}}{m_{1}}\right\rfloor}{\left\lceil\frac{m_{2}}{m_{1}}\right\rfloor}\right\rfloor
\end{aligned}
$$


It should be noted that when $\frac{m_{2}}{m_{1}}, \frac{n}{m_{1}}$, and $\frac{n}{m_{2}}$ are integers, the conditions given in Theorems 1 and 2 reduce to the result proved in our previous study [19], i.e., $k_{\mathrm{MA} 1}=k_{\mathrm{MA} 2}=k \geqslant n$.

The proposed algorithms and derived conditions can be extended to the $r \times r$ switching fabrics. In the following, we show how this can be conducted in the case when $r=4$. The connection matrix:

$\mathbf{H}^{m_{x}}=\left[\begin{array}{llll}h_{11}^{m_{x}} & h_{12}^{m_{x}} & h_{13}^{m_{x}} & h_{14}^{m_{x}} \\ h_{21}^{m_{x}} & h_{22}^{m_{x}} & h_{23}^{m_{x}} & h_{24}^{m_{x}} \\ h_{31}^{m_{x}} & h_{32}^{m_{x}} & h_{33}^{m_{x}} & h_{34}^{m_{x}} \\ h_{41}^{m_{x}} & h_{42}^{m_{x}} & h_{43}^{m_{x}} & h_{44}^{m_{x}}\end{array}\right]$

can be divided into four submatrices in the following manner:

$\mathbf{H}_{\mathbf{1 1}}^{\boldsymbol{m}_{x}}=\left[\begin{array}{ll}h_{11}^{m_{x}} & h_{12}^{m_{x}} \\ h_{21}^{m_{x}} & h_{22}^{m_{x}}\end{array}\right], \mathbf{H}_{\mathbf{1 2}}^{\boldsymbol{m}_{\boldsymbol{x}}}=\left[\begin{array}{ll}h_{13}^{m_{x}} & h_{14}^{m_{x}} \\ h_{23}^{m_{x}} & h_{24}^{m_{x}}\end{array}\right]$,

$\mathbf{H}_{\mathbf{2 1}}^{\boldsymbol{m}_{x}}=\left[\begin{array}{ll}h_{31}^{m_{x}} & h_{32}^{m_{x}} \\ h_{41}^{m_{x}} & h_{42}^{m_{x}}\end{array}\right], \mathbf{H}_{\mathbf{2 2}}^{\boldsymbol{m}_{x}}=\left[\begin{array}{ll}h_{33}^{m_{x}} & h_{34}^{m_{x}} \\ h_{43}^{m_{x}} & h_{44}^{m_{x}}\end{array}\right]$.

The connections represented by matrices $\mathbf{H}_{11}^{\boldsymbol{m}_{\boldsymbol{x}}}$ and $\mathbf{H}_{\mathbf{2}}^{\boldsymbol{m}_{\boldsymbol{x}}}$ (marked in gray in (21)) are not in conflict in the interstage links, so they can use FSUs with the same sequence numbers, and this also applies to the connections in $\mathbf{H}_{\mathbf{1 2}}^{m_{x}}$ and $\mathbf{H}_{22}^{\boldsymbol{m}_{x}}$. The number of FSUs required is then two times the value given by Eqs. (13) or (17), depending on the algorithm employed. This approach can also be used in the case when $r=3$, by simply assuming that input 4 and output 4 are added, and in addition to the original requests, we have also requests between them. Therefore, we can simply conclude that the number of FSUs required when using MA1 is: $k_{\mathrm{MA} 1}^{r}=\lceil r / 2\rceil \cdot k_{\mathrm{MA} 1}^{2}$ and when using MA2, we obtain: $k_{\mathrm{MA} 2}^{r}=\lceil r / 2\rceil \cdot k_{\mathrm{MA} 2}^{2}$, where $k_{\mathrm{MA} 1}^{2}$ is given by (13) and $k_{\mathrm{MA} 2}^{2}$ is given by (17).

\section{Comparisons}

In Theorems 1 and 2, we proved two upper bounds for the RNB conditions under two merging algorithms in $2 \times 2$ WSW1 switching fabrics that serve connections with two rates. Next, we need to determine which algorithm performs better, i.e., the algorithm that needs fewer FSUs in the interstage links. Let us consider the switching fabric with $n=160$ and 320 (these are practical values for the number of FSUs in the C-band of the optical fiber spectrum). For each case, we considered three values of $m_{1}(2,3$, and 4$)$ and set the range for $m_{2}$ from $m_{1}+1$ to 20 .

The number of FSUs required for MA1 and MA2 are plotted in Fig. 8 (for $n=160$ ) and Fig. 9 (for $n=320$ ).

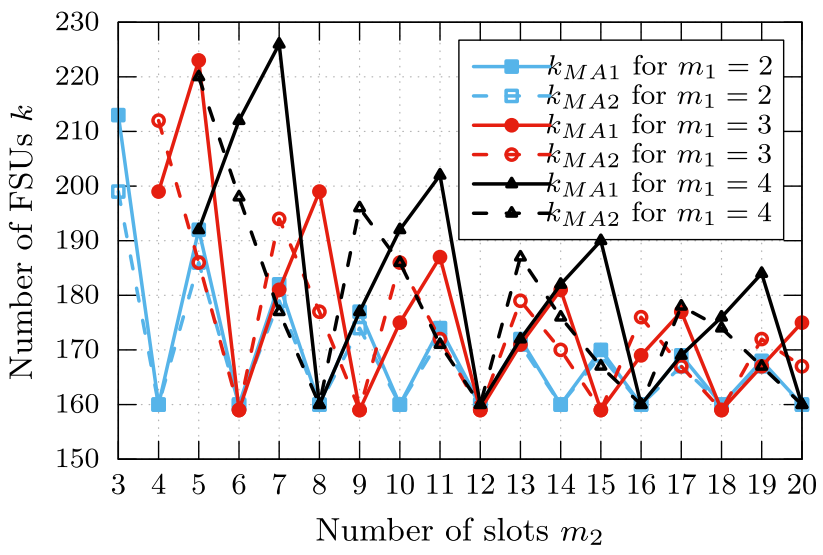

Fig. 8 Number of FSUs $k$ versus $m_{2}$ for $n=160$

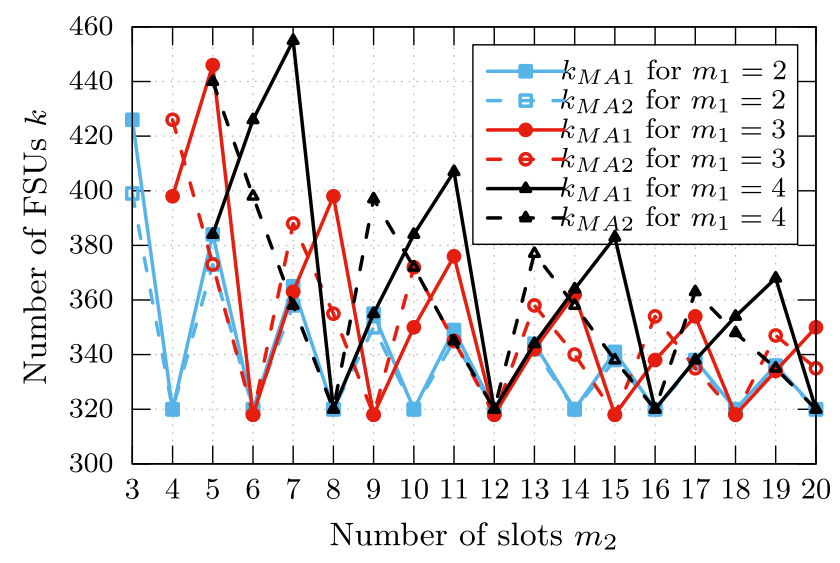

Fig. 9 Number of FSUs $k$ versus $m_{2}$ for $n=320$

These plots indicate that no general relationship shows when MA1 is better than MA2, or vice versa. For instance, for $n=160$ and $m_{1}=2$, MA2 requires no more FSUs than MA1 $\left(k_{\mathrm{MA} 2} \leqslant k_{\mathrm{MA} 1}\right)$ in the considered range of $m_{2}$. For $m_{1} \geqslant 3$, the relationship changes as follows. For $m_{1}=3$ and $m_{2}=4$ we have $k_{\mathrm{MA} 1}=199<k_{\mathrm{MA} 2}=212$, but when $m_{2}=5$ the relationship changes to $k_{\mathrm{MA} 1}=223>k_{\mathrm{MA} 2}=186$. When $m_{2}=6$, we have $k_{\mathrm{MA} 1}=k_{\mathrm{MA} 2}=159$. When $m_{2}$ is increased further, the number of required FSUs required is sometimes better with MA1 and sometimes with MA2, and these numbers are also equal in some cases.

A similar relationship can be observed for $n=320$ (Fig. 9). For $m_{1}=2$, MA2 is always no worse than MA1 for the considered range of $m_{2}$. When $m_{1}>2$, for each combination of connection sizes $m_{1}$ and $m_{2}$, we must check the algorithm that requires fewer FSUs in the interstage links. It should be noted that parameters, such as $n, m_{1}$, and $m_{2}$ are usually known at the design stage for the switching fabric and the optical network because they define the connection requests that can be served in the network. 
The results obtained in this study cannot be compared in a fair manner with the SSNB conditions proposed previously [12] because they were derived for all connection rates from 1 to $m_{\max }$, and thus they are not valid for two arbitrary connection rates. Similarly, the RNB conditions proposed by [19] are only valid when $\frac{m_{2}}{m_{1}}, \frac{n}{m_{1}}$, and $\frac{n}{m_{2}}$ are integers. At present, we are not aware of any other RNB conditions published in other studies.

\section{Conclusions}

In this study, we proposed two versions of merging algorithms that can be used for simultaneous connections routing in $2 \times 2$ WSW1 switching fabrics that serve connections with two rates: $m_{1}$ and $m_{2}$. We did not impose any restrictions on the relationship between $m_{1}, m_{2}$, and $n$ unlike our previous study [19]. When $\frac{m_{2}}{m_{1}}, \frac{n}{m_{1}}$, and $\frac{n}{m_{2}}$ are integers, the proposed RNB conditions reduce to the known results proved in the previous study by [19]. Depending on $m_{1}$ and $m_{2}$, different merging algorithms (MA1 or MA2) may yield better results and less FSUs might be required in the interstage links.

For example, the switching fabric considered in this study can be used in DCNs that employ 2-slot and 3-slot connections are employed, where the former can provide connections with speed of $100 \mathrm{~Gb} / \mathrm{s}$ whereas the latter can provide a transmission speed of $400 \mathrm{~Gb} / \mathrm{s}$ [25]. When $10 \mathrm{FSUs}$ can be used by one Top-of-Rack (ToR) router (i.e., two times 2-slot and two times 3-slot connections can be set up), the DCN can serve a data center comprising up to 64 racks when 320 FSUs are used in the C-band of the optical spectrum. In the case when five FSUs are assigned to one ToR router, the number of connected racks can be increased to 128 .

The proposed results give the upper bound for the RNB conditions which are close to the lower bound results for $n$ provided by [19]. The proposed algorithms can be extended for use in switching fabrics with higher connection rates. However, the number of ways in which the partial permutations can be merged increases rapidly and it is difficult to derive mathematical formules for the required number of FSUs. We will address this problem in future research, as well as testing other approaches for representing the set of connection requests and algorithms for routing them. In this study, we demonstrated how this model can be used for $r \times r$ switching fabrics with two connection rates. It should be noted that this is still the upper bound and the sufficient and necessary conditions still remain open problems.
Acknowledgements The work of Wojciech Kabaciński and Remigiusz Rajewski were supported by the National Science Centre, Poland (NCN) under Grant UMO-2016/21/B/ST7/ 02257 (ERP: 08/ 84/PNCN/2257), and Atyaf Al-Tameemi was supported by funding by the Ministry of Science and Higher Education, Poland.

\section{Compliance with ethical standards}

Conflict of interest The authors declare that they have no conflict of interest.

Open Access This article is distributed under the terms of the Creative Commons Attribution 4.0 International License (http://creativeco mmons.org/licenses/by/4.0/), which permits unrestricted use, distribution, and reproduction in any medium, provided you give appropriate credit to the original author(s) and the source, provide a link to the Creative Commons license, and indicate if changes were made.

\section{References}

1. López, V., Velasco, L.: Elastic Optical Networks. Architectures, Technologies, and Control. Springer, Basel (2016)

2. Jinno, M., Takara, H., Kozicki, B., Tsukishima, Y., Sone, Y., Matsuoka, S.: Spectrum-efficient and scalable elastic optical path network: architecture, benefits, and enabling technologies. IEEE Commun. Mag. 47(November), 66-73 (2009)

3. Gerstel, O., Jinno, M., Lord, A., Yoo, S.J.B.: Elastic optical networking: a new dawn for the optical layer? IEEE Commun. Mag. 50(February), S12-S20 (2012)

4. ITU-T Recommendation G.694.1. Spectral grids for WDM applications: DWDM frequency grid. In: International Telecommunication Union-Telecommunication Standardization Sector (ITU-T) (2012)

5. Tomkos, I., Azodolmolky, S., Sole-Pareta, J., Careglio, D., Palkopoulou, E.: A tutorial on the flexible optical networking paradigm: state of the art, trends, and research challenges. Proc. IEEE 102(9), 1317-1337 (2014)

6. Proietti, R., Liu, L., Scott, R.P., Guan, B., Qin, C., Su, T., Giannone, F., Yoo, S.J.B.: 3D elastic optical networking in the temporal, spectral, and spatial domains. IEEE Commun. Mag. 53(2), 79-87 (2015)

7. Zhang, P., Li, J., Guo, B., He, Y., Chen, Z., Wu, H.: Comparison of node architectures for elastic optical networks with waveband conversion. China Commun. 8, 77-87 (2013)

8. Chen, Y., Li, J., Zhu, P., Niu, L., Xu, Y., Xie, X., He, Y., Chen, Z.: Demonstration of petabit scalable optical switching with subband-accessibility for elastic optical networks. In: Opto Electronics and Communication Conference and Australian Conference on Optical Fibre Technology, OECC/ACOFT 2014 , no. July, Melbourne, Australia, pp. 350-351 (2014)

9. Danilewicz, G., Kabaciński, W., Rajewski, R.: Strict-sense nonblocking space-wavelength-space switching fabrics for elastic optical network nodes. J. Opt. Commun. Netw. 8(10), 745-756 (2016)

10. Kabaciński, W., Michalski, M., Rajewski, R.: Strict-sense nonblocking W-S-W node architectures for elastic optical networks. J. Lightw. Technol. 34(13), 3155-3162 (2016) 
11. Chatterjee, B.C., Sarma, N., Oki, E.: Routing and spectrum allocation in elastic optical networks: a tutorial. IEEE Commun. Surv. Tut. 17(3), 1776-1800 (2015)

12. Kabaciński, W., Michalski, M., Abdulsahib, M.: The strict-sense nonblocking elastic optical switch. In: IEEE 15th Interconnection Conference on High Performance Switching and Routing (HSPR). Budapest, Hungary (July 2015)

13. Beneš, V.E.: Mathematical Theory of Connecting Networks and Telephone Traffic. Academic Press, Cambridge (1965)

14. Kabaciński, W.: Nonblocking Electronic and Photonic Switching Fabrics. Springer, Berlin (2005)

15. Clos, C.: A study of non-blocking switching networks. Bell Syst. Tech. J. 32(2), 406-424 (1953)

16. Hwang, F.: The Mathematical Theory of Nonblocking Switching Networks, vol. 15, 2nd edn. World Scientific Publishing Co., Inc., Hackensack (2004)

17. Jajszczyk, A.: Rearrangeable switching networks composed of digital symmetrical matrices. In: Proceedings on IEEE Mediteranean Electrotechnical Conference, Athens, Greece, p. paper A4.11 (1983)

18. Melen, R., Turner, J.S.: Nonblocking multirate networks. SIAM J. Comput. 18(2), 301-313 (1989)

19. Kabaciński, W., Rajewski, R., Al-Tameemi, A.: Simultaneous connections routing in $\mathrm{W}-\mathrm{S}-\mathrm{W}$ elastic optical switches with limited number of connection rates. In: 21th International Conference on Optical Networks Design and Modeling, Budapest: IFIP, pp. 1-6. (2017)

20. Tsao-Wu, N.T.: On Neiman's algorithm for the control of rearrangeable switching networks. IEEE Trans. Commun. COM22(6), 737-742 (1974)

21. Jajszczyk, A.: A simple algorithm for the control of rearrangeable switching networks. IEEE Trans. Commun. COM-33(2), 169-171 (1985)

22. Cardot, C.: Comments on "A simple algorithm for the control of rearrangeable switching networks". IEEE Trans. Commun. COM-34(4), 395-395 (1986)

23. Barroso, L., Hölzle, U.: The Datacenter as a Computer: An Introduction to the Design of Warehouse-Scale Machines. Morgan \& Claypool, San Rafael (2009)

24. Yoo, S., Yin, Y., Proietti, R.: Elastic optical networking and lowlatency high-radix optical switches for future cloud computing. In: Proceedings on IEEE International Conference on Networking and Communications, pp. 1097-1101 (2013)

25. Zhou, X., Liu, H., Urata, R.: Datacenter optics: requirements, technologies, and trends. Chin. Opt. Lett. 15(5), 120008-1120008-4 (2017)

Publisher's Note Springer Nature remains neutral with regard to jurisdictional claims in published maps and institutional affiliations.

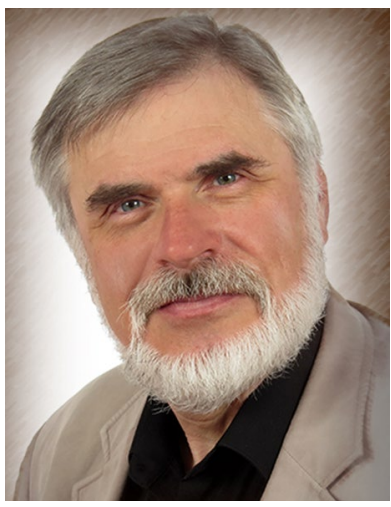

Wojciech Kabaciński is a Professor at Poznan University of Technlology, Poland. He graduated in Telecommunications in 1983 (with honor) from Poznan University of Technology (PUT). In 1988, he received a $\mathrm{Ph} . \mathrm{D}$. degree (his thesis was awarded by the Ministry of National Education), in 1999 he received the Doctor Habilitus degree, both form PUT, and in 2006 he became a full professor. Since 1983, he has been working in the
Institute of Electronics and Telecommunications, Poznan University of Technology, where he currently is a full professor. He worked also as a consultant for telecom industry. He is also a professor of College of Communications and Management. He is the author of the book Nonblocking Electronic and Photonic Switching Fabrics, Springer, 2005, another four books published in Polish, over 140 papers and has 10 patents. His main research interests include: digital switching systems, photonic switching networks and systems, switching network architectures. He served as a reviewer for IEEE Transactions on Communications, IEEE Communications Magazine, IEEE Journal on Selected Areas in Communications, IEEE/ACM Transactions on Networking, IEEE Journal of Lightwave Technology, and Performance Evaluations. He was also one of the Guest Editors of Feature Topic in IEEE Communications Magazine concerning Clos switching networks. He was or currently is a member of technical program committees of international and national conferences and symposia including: IEEE International Conference on Communications 2002, 2004, 2006, IEEE Globecom 2004, 2006, International Conference on Telecommunications (2000, 2001, 2002, 2003, 2004, 2005, and 2006), Polish-German Teletraffic Seminar (2000, 2002, 2004, and 2006), and Conference on Next Generation Internet Design and Engineering NGI 2005 and 2006. He was also the Technical Program Vice-Chair of 2005 Workshop on High Performance Switching and Routing (HPSR 2005), and the General Chair of HPSR 2006, Vice-Chair of IEEE ICC 2009, Co-Chair of symposia organized in ICC 2007 and Globecom 2010. Professor Kabaciński is the senior member of IEEE Communications Society and Association of Polish Electrical Engineers (SEP). In years 2001-2009, he served as the secretary, vice-chair and then the chair of Communications Switching and Routing Technical Committee of Communications Society. In 2001-2007, he was also the chair of IEEE Communications Society Chapter in Poznan.

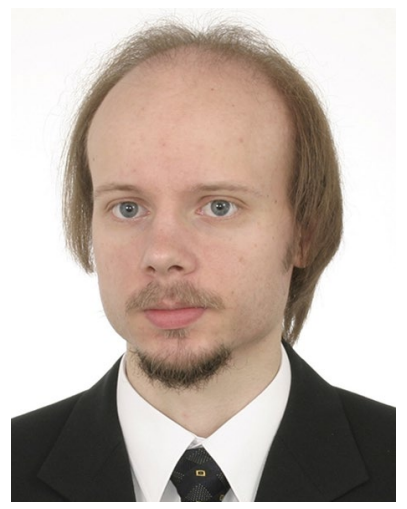

Remigiusz Rajewski received the M.Sc. degree in telecommunications from Poznan University of Technology (PUT), Poland, in 2006. In 2015 he received Ph.D. degree with honors. Since 2010 he has been working at Poznan University of Technology, Faculty of Electronics and Telecommunications, Chair of Communication and Computer Networks. His main research interests include switching fabric architectures, multirate connection in switching fabrics, digital switching systems, photonic switching networks and systems, elastic optical networks, and network security. $\mathrm{He}$ is also interested in: Linux systems (he is Linux Academy instructor) and software $(\mathrm{C}, \mathrm{C}++, \mathrm{C \#}$, and others). He was involved in few ICT projects as: COST Actions, BONE, Future Internet Engineering, and ALIEN. He served as a reviewer for journals: Journal of Lightwave Technology (IEEE/OSA), Journal of Optical Communications and Networking (IEEE/OSA), Journal of Optical Switching and Networking (Elsevier), and Journal of Computer Networks (Elsevier). He served also as a reviewer for the following conferences: International Symposium on Wireless Communication Systems (ISWCS) 2016, 2017, 2018, 2019, IEEE International Conference on Microwave 2017, Network of the Future (NoF) 2017, 2018, IEICE Information and Communication Technology Forum (ICTF) 2014, and International Conference on Telematics and Future Generation Networks (TAFGEN) 2018. He was or currently is a member of technical program committees of several international conferences, including the International Symposium on Wireless Communication Systems (ISWCS) 2016, 2017, 2018, 2019, and Network of the Future (NoF) 2017, 2018. 


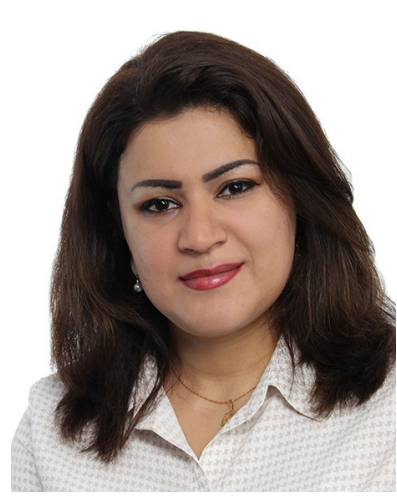

Atyaf Al-Tameemi received a master's degree in Computer Sciences and Communication from Arts, Sciences and Technology University (AUL) in Lebanon in 2012. From 2008 to 2014, she worked in Iraq, in the Computer Science Department at the University of Diyala. Since 2014, she has been a Ph.D. student at Poznan University of Technology (PUT), Faculty of Electronics and Telecommunications, Chair of Communication and Computer Networks. Her main interest is elastic optic networks. 